\title{
Correction to: Agmatine Protects Against 6-OHDA-Induced Apoptosis, and ERK and Akt/GSK Disruption in SH-SY5Y Cells
}

\author{
Esmat Amiri ${ }^{1} \cdot$ Rasoul Ghasemi $^{3,4} \cdot$ Maryam Moosavi $^{1,2}$
}

Published online: 26 July 2018

(c) Springer Science+Business Media, LLC, part of Springer Nature 2018

\section{Correction to: Cell Mol Neurobiol (2016) 36:829-838 https://doi.org/10.1007/s10571-015-0266-7}

The original version of this article unfortunately contained a mistake in the unit of agmatine doses. The agmatine doses were erroneously written in nanomolar in the published article. The correct effective doses of agmatine were 150 and $250 \mu \mathrm{M}$.

The original article can be found online at https://doi.org/10.1007/ s10571-015-0266-7.

Maryam Moosavi

marmoosavi@sums.ac.ir

1 Department of Physiology, School of Medicine, Shiraz University of Medical Sciences, Shiraz, Iran

2 Nanotechnology Research Center, Shiraz University of Medical Sciences, Shiraz, Iran

3 Department of Physiology, Faculty of Medicine, Shahid Beheshti University of Medical Sciences, Tehran, Iran

4 Neurophysiology Research Center, Shahid Beheshti University of Medical Sciences, Tehran, Iran 